\title{
Assessment and Diagnosis of Autism and Spectrum Disorders in Children
}

\author{
Johannes Rojahn • Johnny L. Matson
}

Published online: 22 June 2010

(C) Springer Science+Business Media, LLC 2010

According to the Diagnostic and Statistical Manual of Mental Disorders, 4th ed., text revision (American Psychiatric Association [APA] 2000), Autism Spectrum Disorders (ASD), also known as Pervasive Developmental Disorders (PDD) are neurodevelopmental syndromes that are characterized by pervasive impairments in social interaction and communication, as well as by stereotypic behaviors and restricted interests. They are typically first diagnosed in early childhood and range from severe to a milder form. Diagnostic categories under the ASD umbrella currently include Autistic Disorder, Pervasive Developmental Disorder-Not Otherwise Specified (PDD-NOS), Asperger's Disorder, Childhood Integrative Disorder, and Rett's Disorder. As data from around the world accumulate nosologic controversies about the validity of those classifications persist. This special issue of the Journal of Developmental and Physical Disabilities, which includes both comprehensive review papers as well as data based papers can be grouped into three categories: (1) identification and assessment of ASD core symptoms, (2) diagnosis and assessment of comorbidity in children with ASD, and (3) assessment of adaptive behavior (play skills and verbalizations) in children with ASD.

The discussion on assessment, diagnosis, and prevalence of ASD core symptoms includes the papers by Saracino and Fombonne (Diagnostic and Assessment Issues in Autism Surveillance and Prevalence), Matson and Horowitz (Methods of Early Diagnosis and Tracking for Autism and Pervasive Developmental Disorder Not Otherwise Specified [PDDNOS]), and Matson and Sipes (Stability of Autism Spectrum Disorders Symptoms over Time). Saracino and Fombonne reviewed epidemiological research of ASD between 1966 and 2010. In light of the stunning increase in reported prevalence rates in most countries the authors conclude that

J. Rojahn $(\bowtie)$

George Mason University, Fairfax, VA, USA

e-mail: jrojahn@gmu.edu

J. L. Matson

Louisiana State University, Baton Rouge, LA, USA

e-mail: johnmatson@aol.com 
some of the accelerated case identification rate likely is explained by changes in case definition and identification, public awareness, social services and policies, and study design variables. However, true changes in the underlying incidence of PDDs cannot be ruled out. Matson and Horowitz focus their discussion on the development of assessment methods for young children. In doing so, they review of the most widely used and intensively scrutinized measures at present (ADI-R, ADOS, CHAT and its variants, and the BISCUIT). Matson and Sipes review the extant literature and summarize what we currently know about ASD symptom stability over time. Ghaziuddin presents a study to determine if the Social and Communication Questionnaire (SCQ), a commonly used screening measure for ASD, can differentiate between the two conditions. While differences were found between the ASD and ADHD groups, the SCQ did not differentiate between the ASD and the mixed ASD+ADHD groups. Nine SCQ questions were found as being especially sensitive in differentiating between the ASD and ADHD groups.

Comorbidity of psychopathology (behavioral and psychiatric conditions) in persons with ASD can be difficult to identify as they can mimic core symptoms of ASD. This is particularly true in cognitively lower functions individuals and in young children with ASD. Three papers of this Special Issue focus on the assessment of concurrent behavioral and psychiatric conditions in children with ASD. These are Lecavalier (Validity of Comorbid Psychiatric Disorders in Youngsters with Autism Spectrum Disorders), Fodstad, Rojahn and Matson (Emergent Comorbidity in At Risk Children with and without Autism Spectrum Disorder-A Cross-Sectional Study), and Sturmey (Assessment of Feeding Problems in Children with Autism Spectrum Disorders). Lecavalier describes a study that examined the manifestation of comorbidity (disruptive behavior, anxiety, and mood disorders) in children with ASD as a function of IQ and language skills. Higher functioning children with ASD (IQ<70) were more likely to have a Generalized Anxiety Disorder while nonverbal individuals were more likely to have Oppositional Defiant Disorder. Fodstad, Rojahn and Matson studied developing comorbid psychiatric conditions in toddlers with ASD and compared them to toddlers at risk for developmental delay but without ASD. Toddlers with ASD had more severe comorbid psychiatric symptoms than non-ASD toddlers with an accelerating trend of comorbid behaviors as age increased. This is one of the first studies that points toward an elevated vulnerability of children with ASD for comorbid psychiatric conditions at a very young age as compared to other non-typical populations. Among the many comorbid behavior problems, feeding problems are common among children with ASD. However, the assessment of feeding behavior in this population has received little attention. Sturmey discusses the most promising psychometric assessment instruments of feeding problems in children and adolescents with ASD, including the Brief Autism Mealtime Behavior Inventory (BAMBI) and the Parent Mealtime Action Scale (PMAS). Sturmey suggests that the current definition for pediatric feeding problems in Diagnostic and Statistical Manual of Mental Disorders (American Psychiatric Association [APA] 2000) is limited and incomplete when assessing feeding problems in children with ASD and he proposes changes to diagnostic criteria that may improve the accuracy of the assessment of feeding problems in this population. 
Finally, Boudreau presents an assessment method for play skills and verbalizations in a study that explored the efficacy of video modeling for teaching play skills to two four-year-old boys with ASD. Video-modeling and reinforcement produced rapid increases in modeled play and scripted verbalizations in both children. However, while generalization and short-term maintenance were achieved by both children, long-term maintenance was effective for one child only.

It is hoped that these eight papers of this Special Issue on the assessment and diagnosis of ASD and concurrent problems in children will contribute to the ongoing debate on the nature of ASD and the assessment of comorbid conditions.

\section{Reference}

American Psychiatric Association [APA]. (2000). Diagnostic and statistical manual of mental disorders, (4th ed., text revision). Washington: American Psychiatric Association [APA]. 